\title{
Bleustein-Gulyaev-Shimizu Surface Acoustic Waves in Two-Dimensional Piezoelectric Phononic Crystals
}

\author{
Jin-Chen Hsu and Tsung-Tsong Wu
}

\begin{abstract}
In this paper, we present a study on the existence of Bleustein-Gulyaev-Shimizu piezoelectric surface acoustic waves in a two-dimensional piezoelectric phononic crystal (zinc oxide, $\mathrm{ZnO}$, and cadmium-sulfide, CdS) using the plane wave expansion method. In the configuration of $\mathrm{ZnO}(100) / \mathrm{CdS}(100)$ phononic crystal, the calculated results show that this type of surface waves has higher acoustic wave velocities, high electromechanical coupling coefficients, and larger band gap width than those of the Rayleigh surface waves and pseudosurface waves. In addition, we find that the folded modes of the Bleustein-Gulyaev-Shimizu surface waves have higher coupling coefficients.
\end{abstract}

\section{INTRODUCTION}

Similar to the photonic crystals, periodic structures Smade up of two materials with different elastic properties are referred to as the phononic crystals (PCs) [1]. In the past decade, the similarities between photonic crystals and PCs have inspired many new innovations including the confinement of acoustic energy in defect modes [2], the manufacture of high-efficiency acoustic waveguides [3], the enhancement of sound-light interactions [4], and further triggered study on the peculiar characteristics of acoustic wave propagation in periodic elastic composites [5]-[7]. The existence of band gaps and calculation of phononic band structures have been studied using plane wave expansion (PWE) method [8]-[11], multiple scattering theory (MST) [12], [13], transfer-matrix method [14], and finite-difference time domain (FDTD) method [15][17]. Correspondingly, experimental verifications on the existence of phononic band gap of periodic structures, as well as generation and detection of high-frequency acoustic phonon in GaAs/AlGaAs quantum wells and in isotropic and anisotropic media, also have been carried out and been announced [18]-[22].

The first detailed analysis of Rayleigh surface waves and pseudosurface waves in a two-dimensional periodic elastic structure consisting of AlAs circular cylinders forming a square lattice in a GaAs matrix was proposed by Tanaka and Tamura [8]. Phononic crystals made up of piezoelectric materials is a quite important extension be-

Manuscript received November 3, 2005; accepted December 21, 2005.

The authors are with the Institute of Applied Mechanics, National Taiwan University, Taipei 106, Taiwan (e-mail: wutt@ndt.iam.ntu.edu.tw). cause the high-frequency, surface acoustic waves can be excited easily and detected through piezoelectric effect by interdigital transducers (IDT) on the surface of piezoelectric materials. Wu et al. [10] studied the two-dimensional piezoelectric phononic crystal $\left(\mathrm{Bi}_{12} \mathrm{GeO}_{20} / \mathrm{SiO}_{2}\right)$ and calculated the influence of electrical boundary conditions on Rayleigh surface wave and pseudosurface wave. Laude et al. [23] analyzed the full band gap for surface acoustic waves in a piezoelectric phononic crystal composed of a square-lattice, Y-cut lithium niobate with circular void inclusions.

In a homogeneous piezoelectric material, an SH-type surface acoustic wave with no counterpart in a nonpiezoelectric material was discovered simultaneously and independently by Bleustein [24] and Gulyaev [25]. They showed that the nonvanishing piezoelectric coupling factor in a class of transversely isotropic piezoelectric crystal (e.g., crystal symmetry class $6 \mathrm{~mm}$ ) can result in a surface wave with a very simple mechanical displacement and electric potential fields that decay exponentially into the medium. In early 1969, Shimizu et al. [26] theoretically and experimentally demonstrated the existence of such a unique wave in a poled lead zirconate titanate (PZT) ceramic independent of the knowledge of Bleustein [24] and Gulyaev [25] papers. Since then, the follow-up researches and related applications have made the Bleustein-GulyaevShimizu (BGS) wave theory as one of the cornerstones for the modern signal processing and electroacoustic technology $[27]-[29]$.

Among the existing literature investigating the surface acoustic waves, the BGS surface waves in two-dimensional periodic structures have not been studied so far. In this paper, we present a study on the existence of BGS piezoelectric surface acoustic wave in a two-dimensional piezoelectric PC. The acoustic scattering effect in the periodic structure induces novel properties of the piezoelectric surface wave that do not exist on homogeneous crystal. On taking piezoelectric effect into account and with the appropriate arrangement of the constituents of the piezoelectric PC, we can clearly demonstrate the properties of BGS waves in the two-dimensional piezoelectric PC. The general equations of motion for piezoelectric waves in inhomogeneous media are summarized in Section II. In Section III, we studied and discussed the bulk and surface waves in $\mathrm{ZnO} / \mathrm{CdS}$ piezoelectric periodic structure. Some conclusions and remarks also are given. 


\section{Acoustic Waves in Two-Dimensional Piezoelectric PC}

Consider an inhomogeneous, piezoelectric medium occupying the half space $z>0$ with a flat surface $(z=0)$. We assume that this system exhibits spatial periodicity and has translational symmetry in the $z$-direction. This means the material properties of the medium depend only on the coordinate variables $x$ and $y$. In the quasistatic approximation, the governing field equations of piezoelectricity can be expressed as [30]:

$$
\begin{aligned}
\rho \ddot{u}_{j} & =\partial_{i} T_{i j}, \\
\partial_{i} D_{i} & =0,
\end{aligned}
$$

where $\rho(\mathbf{x})$ is the position-dependent mass density, $u_{j}(\mathbf{r}, t)$ is the mechanical displacement vector, $\mathbf{r}=(\mathbf{x}, z)=$ $(x, y, z)$ is the position vector, and $T_{i j}(\mathbf{r}, t)$ and $D_{i}(\mathbf{r}, t)$ are the stress and electric displacement fields, respectively. The piezoelectric constitutive relations with the displacement $u_{j}(\mathbf{r}, t)$ and the electric potential $\phi(\mathbf{r}, t)$ as variables are given by [31]:

$$
\begin{aligned}
T_{i j} & =c_{i j k l} \partial_{l} u_{k}+e_{l i j} \partial_{l} \phi, \\
D_{i} & =e_{i k l} \partial_{l} u_{k}-\varepsilon_{i l} \partial_{l} \phi,
\end{aligned}
$$

where $c_{i j k l}(\mathbf{x}), \quad e_{l i j}(\mathbf{x})$, and $\varepsilon_{i l}(\mathbf{x})$ are the positiondependent elastic stiffness constants, piezoelectric constants, and permittivity, respectively. Due to the spatial periodicity of the structure and applying the Bloch theorem, the material constant $\alpha$, mechanical displacement vector $u_{j}(\mathbf{r}, t)$, and electric potential $\phi(\mathbf{r}, t)$ can be expanded in Fourier series:

$$
\begin{gathered}
\alpha(\mathbf{x})=\sum_{\mathbf{G}} e^{i \mathbf{G} \cdot \mathbf{x}} \alpha_{\mathbf{G}}, \\
\left\{\begin{array}{c}
u_{x}(\mathbf{r}, t) \\
u_{y}(\mathbf{r}, t) \\
u_{z}(\mathbf{r}, t) \\
\phi(\mathbf{r}, t)
\end{array}\right\}=e^{i(\mathbf{k} \cdot \mathbf{x}-\omega t)} \sum_{\mathbf{G}} e^{i \mathbf{G} \cdot \mathbf{x}}\left\{\begin{array}{c}
A_{\mathbf{G}}^{1} \\
A_{\mathbf{G}}^{2} \\
A_{\mathbf{G}}^{3} \\
A_{\mathbf{G}}^{4}
\end{array}\right\} e^{i k_{z} z},
\end{gathered}
$$

where the generalized displacement vector $\mathbf{u}=$ $\left\{u_{x}, u_{y}, u_{z}, \phi\right\}^{T}$ is introduced for convenience. $\mathbf{G}$ and $\mathbf{k}=\left(k_{x}, k_{y}\right)$ are the two-dimensional reciprocal-lattice vector (RLV) and the Bloch wave vector defined in the irreducible surface Brillouin zone, respectively. $\alpha=$ $\left(\rho, c_{i j k l}, e_{l i j}, \varepsilon_{i l}\right)$, and $\alpha_{\mathbf{G}}$ is the corresponding Fourier coefficients that can be calculated by the integral $\alpha_{\mathbf{G}}=$ $A_{\text {cell }}^{-1} \int_{\text {cell }} \alpha(\mathbf{x}) e^{-i \mathbf{G} \cdot \mathbf{x}} d^{2} x . \omega$ is the circular frequency, and $k_{z}$ is the wave number along the $z$-direction. In practice, the summations in (3) and (4) are truncated by choosing $n$ reciprocal-lattice vectors G. Substituting (3) and (4) into (1) and (2), we obtain a generalized eigenvalue problem:

$$
\left(\mathbf{R} k_{z}^{2}+\mathbf{Q} k_{z}+\mathbf{P}\right) \cdot \mathbf{U}=0
$$

where $\mathbf{U}=\left\{\mathbf{A}_{\mathbf{G}^{\prime}}^{1}, \mathbf{A}_{\mathbf{G}^{\prime}}^{2}, \mathbf{A}_{\mathbf{G}^{\prime}}^{3}, \mathbf{A}_{\mathbf{G}^{\prime}}^{4}\right\}^{T}$ is the Fourier component of the generalized displacement vector. The explicit expressions of the $4 n \times 4 n$ matrices $\mathbf{R}, \mathbf{Q}$, and $\mathbf{P}$, which are functions of $\mathbf{k}, \mathbf{G}, \omega$, and $\alpha_{\mathbf{G}}$, can be found in [9] and [10]. Note that the case of piezoelectric bulk waves propagating along the $x-y$ plane in an infinite $\mathrm{PC}$ can be computed using (5) by setting $k_{z}=0$. Solving (5) yields $8 n$ eigenvalues and eigenvectors; but, for a surface wave solution, the mechanical displacement and the electric potential fields exponentially decay into the half space, and thus, the $4 n$ complex wave number $k_{z}$ 's with positive imaginary part are chosen. Accordingly, we put $a_{\mathbf{G}}^{j(l)}=X_{l} a_{\mathbf{G}}^{j(l)},(j=1-4$ and $l=1-4 n)$, in (4) and sum over $l$, where $a_{\mathbf{G}}^{j(l)}$ is the associated eigenvector of the eigenvalue $k_{z}^{(l)}$, and $X_{l}$ is the undetermined weighting coefficient that can be determined from the boundary conditions on the surface $z=0$. The mechanical boundary conditions at free surface require the nullity of stress components $\left.T_{i 3}\right|_{z=0}=\left.\left(c_{i 3 k l} \partial_{l} u_{k}+e_{l i 3} \partial_{l} \phi\right)\right|_{z=0}=0,(1=1,2,3)$. For the electric boundary condition, two important cases are distinguished as follows.

- If the surface is a free surface, we have the open-circuit condition with $\left.D_{3}\right|_{z=0}=\left.\left(e_{3 k l} \partial_{l} u_{k}-\varepsilon_{3 l} \partial_{l} \phi\right)\right|_{z=0}=$ $-\left.\varepsilon_{0}\left(\partial_{3} \phi_{\text {air }}\right)\right|_{z=0}$, giving the phase velocity $V=V_{0}$. Note that the electric potential in the air can be solved by the Laplace equation $\nabla^{2} \phi_{\text {air }}=0$ together with the boundary conditions $\left.\phi\right|_{z=0}=\left.\phi_{a i r}\right|_{z=0}$ and $\left.\phi_{\text {air }}\right|_{z \rightarrow-\infty}=0$.

- If the surface is covered by a very thin metallic film, we have the short-circuit condition with $\left.\phi\right|_{z=0}=0$, giving the phase velocity $V=V_{m}$.

These boundary conditions lead to a homogeneous linear system of equations for $X_{l}$ :

$$
\mathbf{H} \cdot\left(\begin{array}{c}
X_{1} \\
X_{2} \\
\vdots \\
X_{4 n}
\end{array}\right)=\mathbf{0},
$$

where $\mathbf{H}$ is a $4 n \times 4 n$ matrix. For the existence of a nontrivial solution of $X_{l}$, the following condition must be satisfied:

$$
\operatorname{det}(\mathbf{H})=0 .
$$

Eq. (6) and (8) should be solved simultaneously to determine the dispersion relation for the surface waves. By using the well-known Ingebrigtsen approximation [32], the electromechanical coupling coefficient of a piezoelectric medium can be approximated as $K^{2}=2\left(V_{0}-V_{m}\right) / V_{0}$.

\section{BGS Surface Waves in a ZnO(100)/CDS(100) Piezoelectric PC}

\section{A. Arrangement of the Constituents}

To develop a numerical example, we consider the piezoelectric $\mathrm{PC}$ in which the circular $\mathrm{ZnO}$ cylinders (denoted by $A$ ) embedded periodically in the CdS matrix (denoted 
TABLE I

Material Constants Used in the Calculation.

\begin{tabular}{|c|c|c|c|c|c|c|c|c|c|c|c|}
\hline \multirow[b]{2}{*}{ Material } & \multicolumn{5}{|c|}{$\begin{array}{c}\text { Stiffness } \\
\left(10^{10} \mathrm{~N} / \mathrm{m}^{2}\right) \\
\end{array}$} & \multicolumn{3}{|c|}{$\begin{array}{c}\text { Piezoelectric constant } \\
\left(\mathrm{C} / \mathrm{m}^{2}\right) \\
\end{array}$} & \multicolumn{2}{|c|}{$\begin{array}{l}\text { Permittivity } \\
\left(10^{-11} \mathrm{~F} / \mathrm{m}\right)\end{array}$} & \multirow{2}{*}{$\begin{array}{c}\begin{array}{c}\text { Mass } \\
\text { density } \\
\left(\mathrm{kg} / \mathrm{m}^{3}\right)\end{array} \\
\rho\end{array}$} \\
\hline & $c_{11}$ & $c_{12}$ & $c_{13}$ & $c_{33}$ & $c_{44}$ & $e_{15}$ & $e_{31}$ & $e_{33}$ & $\varepsilon_{11}$ & $\varepsilon_{33}$ & \\
\hline $\mathrm{CdS}$ & 8.56 & 5.32 & 4.62 & 9.36 & 1.49 & -0.21 & -0.24 & 0.44 & 7.99 & 8.44 & 4824 \\
\hline $\mathrm{ZnO}$ & 20.97 & 12.11 & 10.51 & 21.09 & 4.25 & -0.59 & -0.61 & 1.14 & 7.38 & 7.83 & 5676 \\
\hline
\end{tabular}

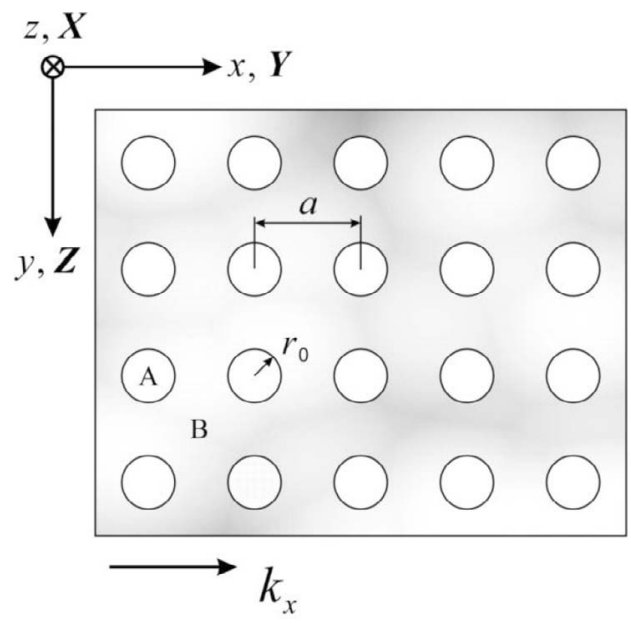

(a)

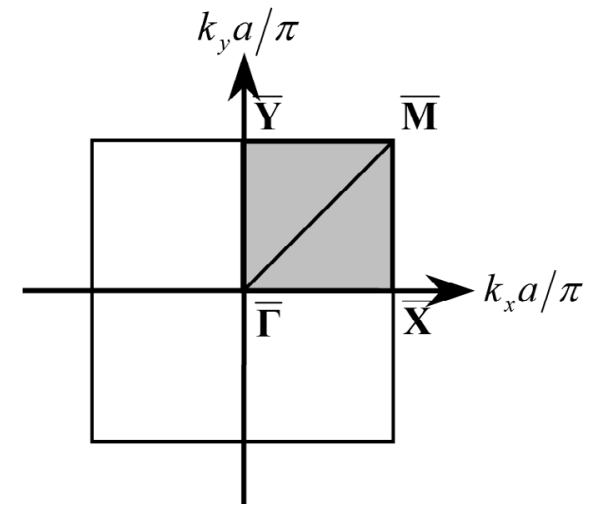

(b)

Fig. 1. (a) A schematic of the two-dimensional piezoelectric PC and the arrangement of the constituents. (b) The corresponding extended irreducible surface Brillouin zone $\overline{\mathbf{\Gamma X M Y \Gamma}}$. $(\mathbf{X}, \mathbf{Y}, \mathbf{Z})$ are the crystallographic axes to describe the orientations of $\mathrm{ZnO}$ and $\mathrm{CdS}$. Note that the $\mathbf{Z}$-axis (crystallographic $c$-axis of $\mathrm{ZnO}$ and $\mathrm{CdS}$ ) coincides with the $y$-axis, and the structure occupies the half-space $z>0$.

by $B$ ) forming an infinite square lattice with lattice spacing $a$ [see Fig. 1(a)]. In this structure, the reciprocal-lattice vectors are $\mathbf{G}=2 \pi\left(n_{1} / a, n_{2} / a\right)$ with integer $n_{1}$ and $n_{2}$, and the filling fraction is $f=\pi r_{0}^{2} / a^{2}=0.3$, where $r_{0}$ is the radius of the cylinders. The Fourier coefficients for this structure are given by:

$$
\alpha_{\mathbf{G}}= \begin{cases}f \alpha_{A}+(1-f) \alpha_{B} & \text { for } \mathbf{G}=0 \\ \left(\alpha_{A}-\alpha_{B}\right) F(\mathbf{G}) & \text { for } \mathbf{G} \neq 0\end{cases}
$$

where $F(\mathbf{G})=2 f J_{1}\left(G r_{0}\right) /\left(G r_{0}\right)$ with $J_{1}\left(G r_{0}\right)$ being the first kind Bessel function of order 1 [33]. In the arrange-

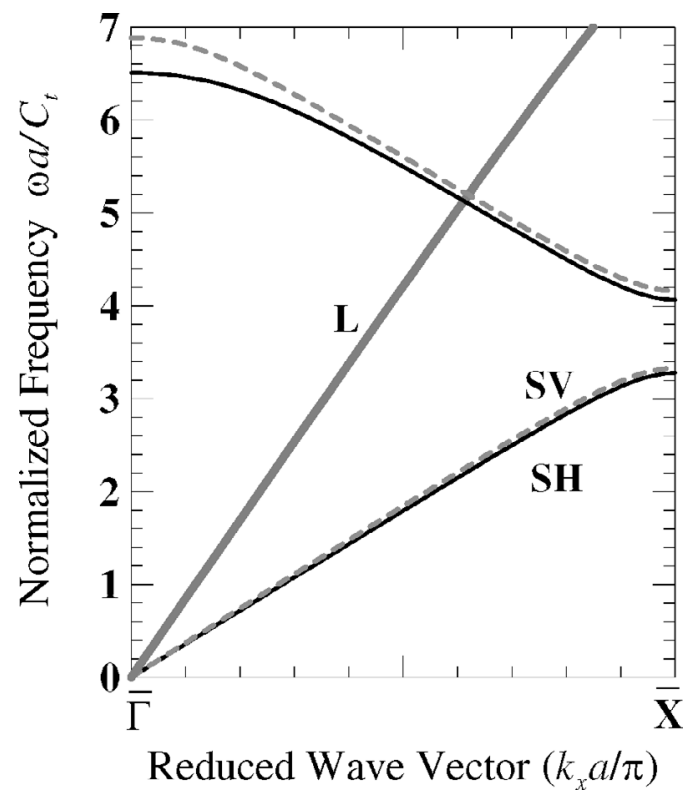

Fig. 2. Acoustic band structure of bulk longitudinal (L, bold solid line), shear vertical (SV, dashed lines), and shear horizontal ( $\mathrm{SH}$, thin solid lines) waves propagating in the infinite piezoelectric PC along $\overline{\boldsymbol{\Gamma}}-\overline{\mathbf{X}}$ direction (where $C_{t}=\sqrt{c_{44}^{B} / \rho^{B}}=1757 \mathrm{~m} / \mathrm{s}$ )

ment of the constituents of the piezoelectric PC, we set the crystal plane (100) of both the piezoelectric materials $\mathrm{ZnO}$ and $\mathrm{CdS}$ to be parallel to the $x-y$ plane, and the six-fold symmetry axis (Z-axis) of the materials coincides with the $y$-axis of the PC so that the piezoelectric effect can compensate for the mechanical displacements to satisfy the stress-free boundary condition on the surface [34], and the SH component of displacement amplitude of acoustic waves could decay into the medium. Fig. 1(b) shows the irreducible surface Brillouin zone for the $\mathrm{ZnO}(100) / \mathrm{CdS}(100)$ piezoelectric PC. The extension of the irreducible Brillouin zone from conventional triangle $\overline{\boldsymbol{\Gamma X M} \boldsymbol{\Gamma}}$ to square $\overline{\boldsymbol{\Gamma X M Y \Gamma}}$ is necessary due to the crystalline anisotropies of $\mathrm{ZnO}(100)$ and $\mathrm{CdS}(100)$ [9].

\section{B. Calculated Results and Discussions}

Fig. 2 displays the acoustic band structure (i.e., the dispersion curves) of bulk waves propagating in the infinite piezoelectric phononic crystal along the $\overline{\mathbf{\Gamma}}-\overline{\mathbf{X}}$ direction. There exists frequency gaps of the bulk shear horizontal (in-plane polarization) and shear vertical ( $z$-direction polarization) waves. Material constants used in the cal- 


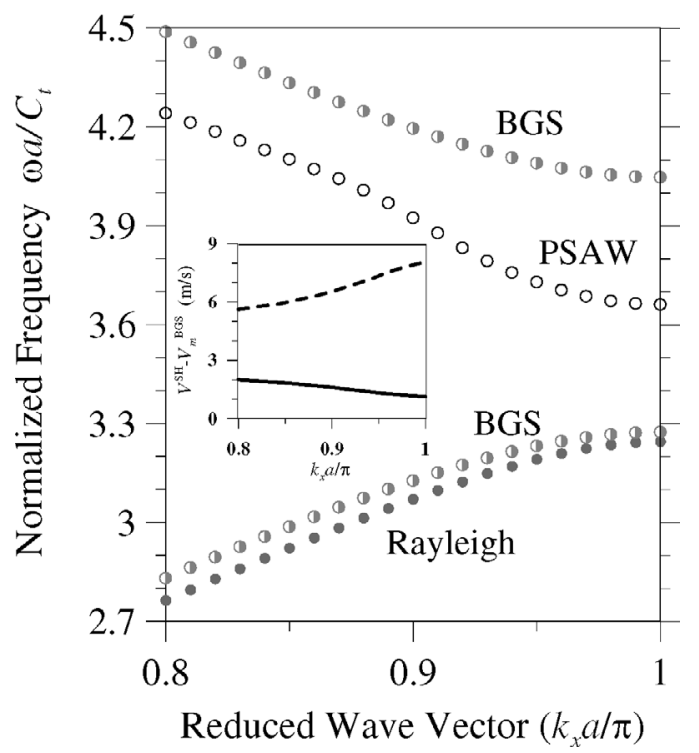

Fig. 3. The details of the dispersion relations of Rayleigh surface wave (dots), pseudosurface wave (open circles), and BGS surface wave (half-filled circles) under shorted boundary condition in the frequency range around the band gap near the $\mathbf{X}$ point. The inset shows the differences in velocity between the piezoelectric bulk $\mathrm{SH}$ modes and the BGS surface modes under shorted boundary condition. The solid line represents the differences between fundamental branches and the dashed line represents the differences between folded branches.

culation are listed in Table I [30]. In all of the calculations, we used 49 RLVs $\left(n_{1}, n_{2}=0, \pm 1, \pm 2, \pm 3\right)$. The convergence test shows satisfactory accuracy for the lowfrequency part. Fig. 3 shows details of the dispersion relations of surface waves under shorted boundary conditions in the frequency range around the band gap near the $\overline{\mathbf{X}}$ point. In Fig. 3, the true Rayleigh-type surface wave branch (dots) exists well below the piezoelectric bulk SH mode. In addition to the Rayleigh surface wave, there exists another branch of surface wave solution (half-filled circles) between the Rayleigh surface wave and the piezoelectric bulk SH mode for this piezoelectric phononic crystal. Although this surface wave branch is very close to the piezoelectric bulk SH mode, the difference still can be distinguished clearly. Further calculation will show this surface wave branch is mainly composed of shear horizontal component, that is a BGS surface wave in the twodimensional piezoelectric $\mathrm{PC}$, and it degenerates into the bulk SH wave as the piezoelectricity is ignored. Moreover, the folded branch of the BGS surface wave has been found in the calculations, the folded BGS surface wave is slightly below the folded bulk SH wave branch and higher than the pseudosurface wave branch. This folded BGS branch with very small attenuation is also a pseudosurface type of wave. The differences in velocity between the piezoelectric bulk SH modes and the BGS surface modes under shorted boundary condition are displayed in the inset of Fig. 3, in which the solid line represents the differences between fundamental branches and the dashed line represents the differences between folded branches. The band gap width of BGS waves is $\Delta \omega a / C_{t}=0.773$. To obtain
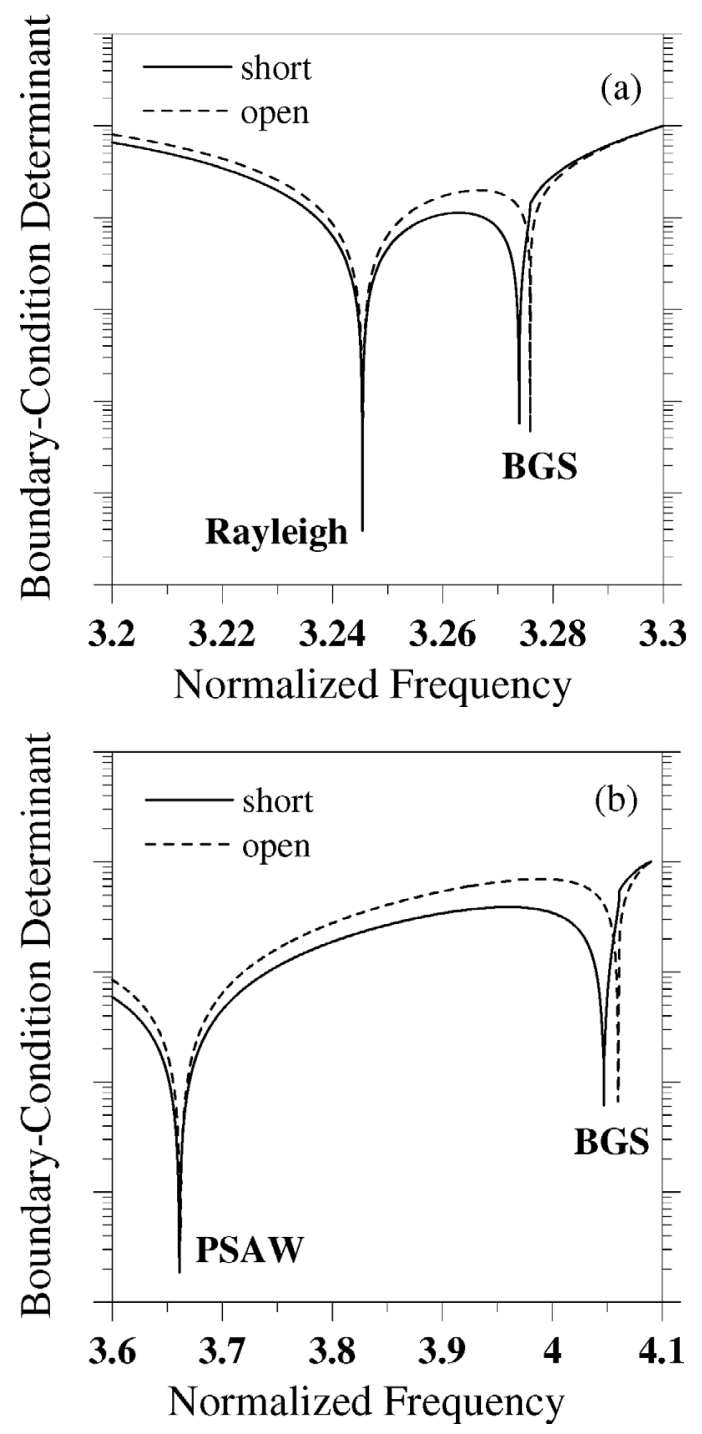

Fig. 4. Short-circuit (solid lines) and open-circuit (dashed lines) boundary-condition determinants for the $\overline{\mathbf{X}}$ point. (a) The frequency range is around the fundamental modes of surface waves. (b) The frequency range is around the folded modes of surface waves.

accurate pseudosurface wave solutions, we added a small, positive imaginary part into the two-dimensional wave vector and assigned, at the same time, a negative imaginary part to one or two $k_{z}$ 's of the dominant partial waves in the calculation such that the surface wave becomes attenuated along the propagation direction to balance the acoustic energy radiating into the bulk of the PC [8].

To reveal the influence of the electrical boundary conditions on the BGS surface wave in the piezoelectric PC, a detailed analysis for the symmetry point $\overline{\mathbf{X}}$ has been conducted. Figs. 4(a) and (b) display the boundary condition determinants of the fundamental and the folded branches as a function of frequency, respectively. In both figures, solid lines denote short-circuit boundary conditions, and dashed lines denote open-circuit boundary conditions. In the case of the fundamental branch [Fig. 4(a)], there are two sharp minima for each boundary-condition determinant. The first one with lower frequency indicates 

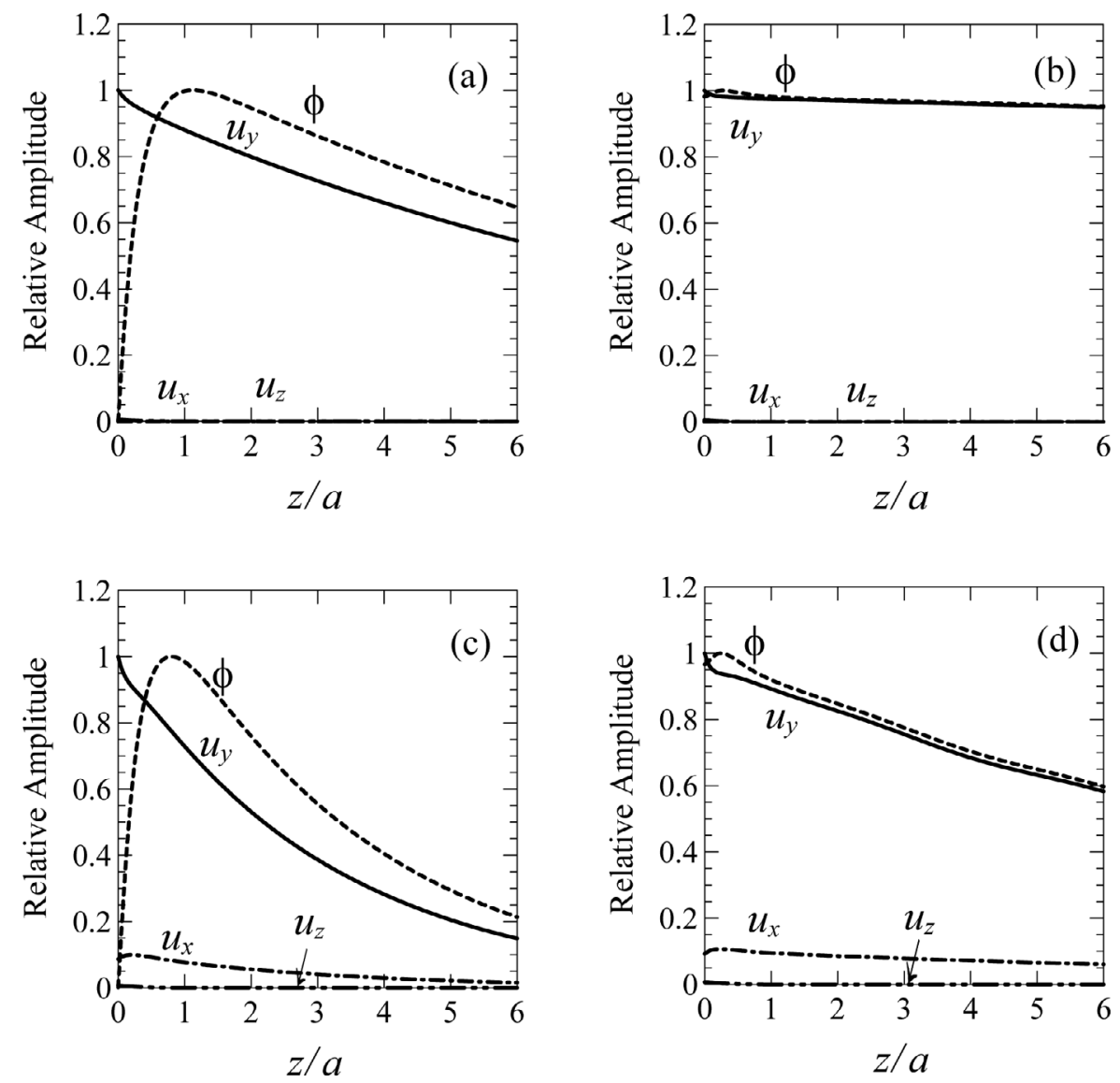

Fig. 5. Displacement fields and electrical potentials of the BGS surface waves at the center of the cylinder versus distance from the surface. (a) The fundamental mode for short-circuit condition. (b) The fundamental mode for open-circuit condition. (c) The folded mode for short-circuit condition. (d) The folded mode for open-circuit condition.

the Rayleigh surface wave, and the one with higher frequency denotes the BGS surface wave. In the case of the Rayleigh wave, we found that the frequency difference between the open-circuit and short-circuit conditions is very small. In other words, the electromechanical coupling coefficient is almost vanished for the Rayleigh surface wave. For the BGS surface waves, there is a clear difference between the opened and the shorted boundary conditions, and the resulted electromechanical coupling coefficient is about $0.12 \%$. Shown in Fig. 4(b) are similar results for the folded branches, except that the first sharp minimum indicates the pseudosurface wave. The electromechanical coupling coefficient of the folded BGS surface wave is $0.63 \%$. In this piezoelectric PC, the BGS surface waves are considerably more sensitive to the electrical boundary conditions than the Rayleigh surface waves.

For a class of transversely isotropic piezoelectric single crystal, the transverse-wave piezoelectric coupling factor $K_{T}=e_{15} / \sqrt{c_{44} \varepsilon_{11}+e_{15}^{2}}$ was introduced to measure the difference of phase velocities between the piezoelectric bulk $\mathrm{SH}$ wave and the BGS surface wave and to evaluate the decay of displacements and electric potential for BGS surface waves [30]. The BGS wave velocities in homogeneous piezoelectric medium are given by the formulas $V_{m}=$ $V_{T} \sqrt{1-K_{T}^{4}}$ and $V_{0}=V_{T} \sqrt{1-\left(K_{T}^{4} /\left(1+\varepsilon_{11} / \varepsilon_{0}\right)^{2}\right)}$, where $V_{T}=\sqrt{\left(c_{44}+\left(e_{15}^{2} / \varepsilon_{11}\right)\right) / \rho}$ is the phase velocity of the piezoelectric bulk transverse wave. And the decay constants $\beta$ along depth are $\beta_{m}=K_{T}^{2}$ and $\beta_{0}=$ $K_{T}^{2} /\left(1+\varepsilon_{11} / \varepsilon_{0}\right)$ for short-circuit and open-circuit conditions, respectively. As the coupling factor $K_{T}$ increases, difference of the phase velocities between piezoelectric bulk transverse wave and BGS surface wave becomes larger, and the decay of the mechanical displacements and electric potential become more rapid. For the case of $\mathrm{CdS}$, $K_{T, \mathrm{CdS}}=0.189, \beta_{m, \mathrm{CdS}}=0.0357, \beta_{0, \mathrm{CdS}}=0.00356$, $V_{m, \mathrm{CdS}}=1788.59 \mathrm{~m} / \mathrm{s}$, and $V_{0, \mathrm{CdS}}=1789.72 \mathrm{~m} / \mathrm{s}$. For the case of $\mathrm{ZnO}, K_{T, \mathrm{ZnO}}=0.316, \beta_{m, \mathrm{ZnO}}=0.0999$, $\beta_{0, \mathrm{ZnO}}=0.0107, V_{m, \mathrm{ZnO}}=2869.79 \mathrm{~m} / \mathrm{s}$, and $V_{0, \mathrm{ZnO}}=$ $2884.04 \mathrm{~m} / \mathrm{s}$. In the PC, these parameters can be comprehended from the depth dependence of the displacement fields. In Fig. 5, we plot the depth dependence of the displacement and electric potential fields of the BGS surface waves at the center of the cylinder at point $\overline{\mathbf{X}}$ in the piezoelectric PC. Figs. 5(a) and (b) show the profiles of the displacement amplitudes of the fundamental modes of the BGS surface waves under short circuit and open circuit conditions, respectively. The results show that compared with the shear horizontal component, the other two components are negligible. In addition, the decay of the displacements and the electric potential is much more rapid 
for the short-circuit condition than that of the open-circuit condition. From the dispersion relations of the piezoelectric $\mathrm{PC}$, the phase velocities of fundamental BGS surface modes with reduced wave vector $k a / \pi$ at symmetry point $\overline{\mathbf{X}}$ are $V_{m, \mathrm{PC}}^{1}=1830.97 \mathrm{~m} / \mathrm{s}$ and $V_{0, \mathrm{PC}}^{1}=1832.09 \mathrm{~m} / \mathrm{s}$ for short-circuit conditions and open-circuit conditions, respectively, where the superscript 1 means the fundamental BGS mode. Figs. 5(c) and (d) show the profiles of the displacement amplitudes of the folded BGS surface wave at point $\overline{\mathbf{X}}$ under short-circuit and open-circuit conditions, respectively. The results show that the folded BGS surface waves decay faster than the fundamental modes. However, in this branch a nonvanishing, longitudinal component exists. In other words, the BGS surface wave in this twodimensional piezoelectric PC contains polarization components other than the shear horizontal component. This phenomenon is caused by the scattering effect due to the periodic cylindrical fillers in the structure and can be explained by two reasons:

- The shear horizontal vibration $\left(u_{y}\right)$ is coupled with the sagittal-plane vibration $\left(u_{x}\right.$ and $\left.u_{z}\right)$ through the elastic terms in (5) (detail of the matrix elements can be found in the Appendix of [9], [10]).

- The electric potential is coupled with the mechanical vibration (not only with the shear horizontal vibration but also with the sagittal-plane vibration) through the piezoelectric and dielectric terms in (5).

These two effects (i.e., the coupling from scattering effect of the periodic structure and the anisotropic property of the constituents) do not appear in the homogeneous media belonging to 6 -mm crystal in which the shear horizontal vibration with piezoelectricity is decoupled from the sagittal-plane vibration (and the sagittal-plane vibration is piezoelectric inactive) for acoustic waves propagating in the (100)[010] direction. The coupling of $u_{x}$ with shear horizontal vibration is stronger than $u_{z}$; therefore, the longitudinal polarization is larger than the shear vertical polarization, which is very small. The phase velocities of folded mode of the BGS waves with reduced wave vector at symmetry point $\overline{\mathbf{X}}$ are $V_{m, \mathrm{PC}}^{2}=2263.43 \mathrm{~m} / \mathrm{s}$ and $V_{0, \mathrm{PC}}^{2}=2270.60 \mathrm{~m} / \mathrm{s}$. Contrast to the BGS surface wave in a homogeneous piezoelectric material $\mathrm{CdS}$, the BGS surface waves in PC decay a little bit faster for the fundamental mode along the depth and even faster for the folded mode. From the above investigation, we have demonstrated the existence of the BGS surface waves in a two-dimensional piezoelectric PC.

In Fig. 6(a) and (b), we show the band edges of surface wave frequency gaps and their normalized gap widths at the point $\overline{\mathbf{X}}$ as a function of filling fraction $f$. Fig. 6 shows that the BGS surface wave (open circles) band gaps are larger than that between the Rayleigh surface wave and the pseudosurface wave. The maximum band gap appears at $f=0.576$ in this case. Shown in Fig. 7 is variation of the electromechanical coupling coefficients of the BGS surface waves at the $\overline{\mathbf{X}}$ point as a function of filling fraction. The folded mode has higher values than that of fun-
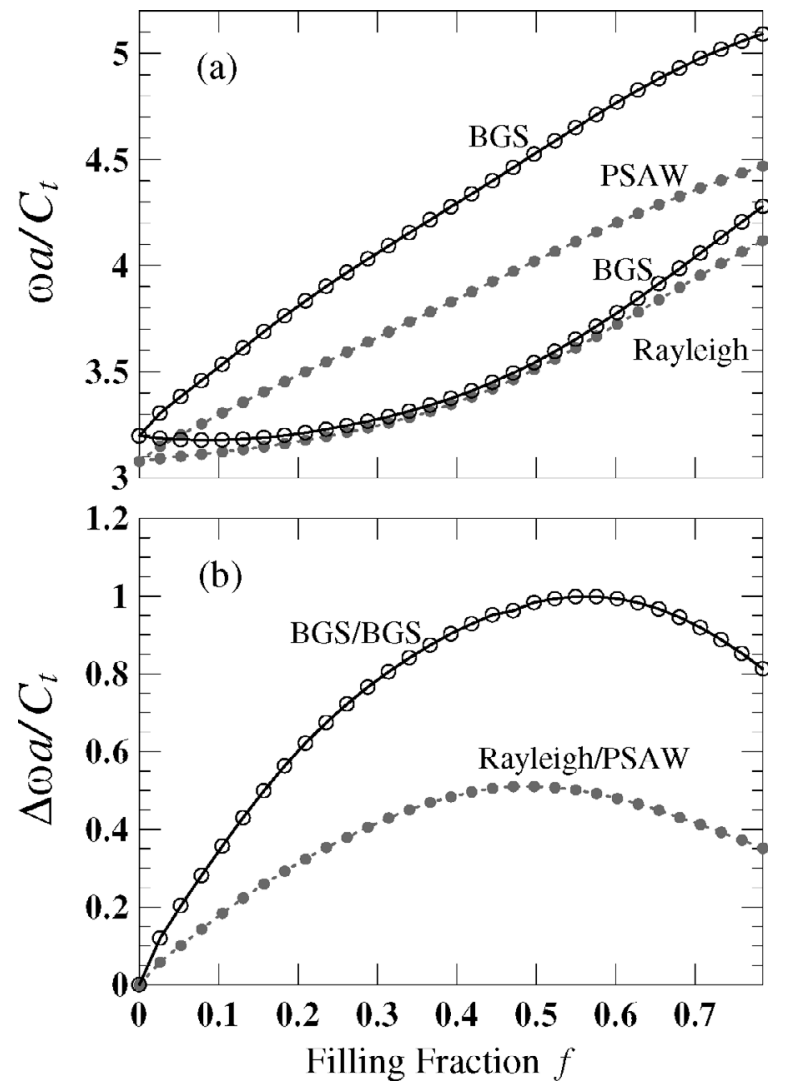

Fig. 6. (a) The band edges of the band gaps. (b) The normalized gap widths for Rayleigh surface waves (dots) and BGS surface waves (open circles) at the $\overline{\mathbf{X}}$ point versus filling fraction $f$.

damental mode, and both increase progressively with the increase of filling fraction. The electromechanical coupling coefficients of $\mathrm{CdS}$ and $\mathrm{ZnO}$ given by Ingebrigtsen approximation are 0.126 and $0.988 \%$, respectively. Comparison of the electromechanical coupling coefficients of PC shown in Fig. 7 with those of $\mathrm{CdS}$ and $\mathrm{ZnO}$ could be drawn that the coupling coefficients of folded mode are considerably increased by the scattering effect in some higher filling fraction range [10]. The above observations also lead to that the frequency gap width and the electromechanical coupling coefficients of the BGS surface wave are larger than those of the Rayleigh surface wave.

\section{Conclusions}

In this paper, we have demonstrated the existence of BGS surface waves in a two-dimensional piezoelectric PC composed of two different hexagonal materials $(\mathrm{ZnO}$ and $\mathrm{CdS}$ ); and we discussed their propagation properties using the PWE method. In this particular configuration of $\mathrm{ZnO}(100) / \mathrm{CdS}(100)$ piezoelectric $\mathrm{PC}$ and in the propagation direction along the $x$-axis (crystallographic orientation $k_{\mathbf{Y}}$ ), theoretical calculations showed that this type of surface wave has no counterpart in a purely elastic PC. Moreover, the results showed that the BGS surface waves have higher acoustic wave velocities, high electromechani- 


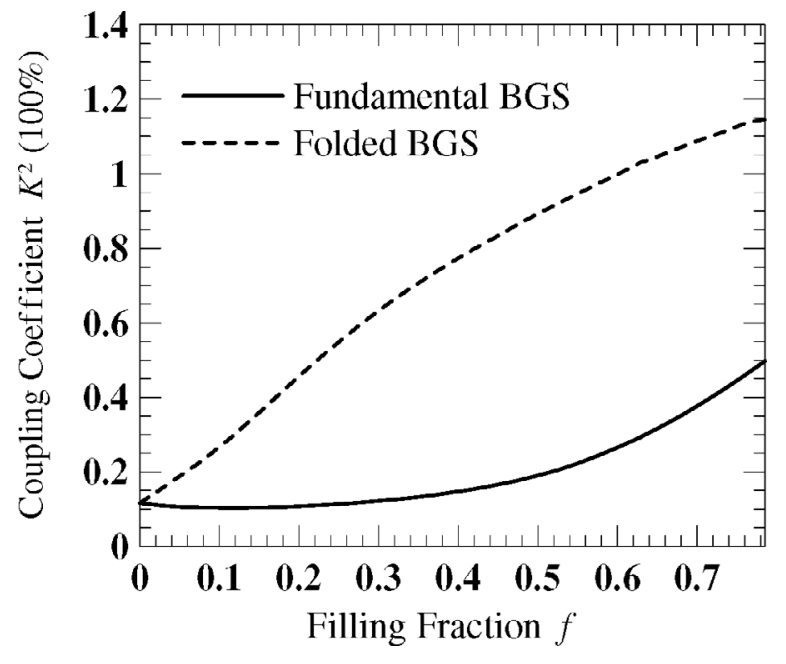

Fig. 7. Electromechanical coupling coefficients of the BGS surface waves calculated along the frequency edges at the $\overline{\mathbf{X}}$ point versus filling fraction $f$.

cal coupling coefficients, and larger band gap width than those of the Rayleigh surface waves. In addition, we find that the folded modes have higher coupling coefficients. In the present calculation, a square lattice is considered for piezoelectric periodic composite consisting of the $\mathrm{ZnO}$ cylinders in the CdS host. However, BGS surface waves can exist in other classes of piezoelectric crystals, such as cubic crystal of 23 and $43 \mathrm{~m}$ classes and orthorhombic crystal of $2 \mathrm{~mm}$ class, the properties of BGS wave propagating in the piezoelectric PC composed of these piezoelectric materials would be worth studying.

\section{ACKNOWLEDGMENT}

The authors gratefully acknowledge the National Science Council of Taiwan for the financial support (Grant No. NSC 94-2212-E-002-040).

\section{REFERENCES}

[1] M. M. Sigalas and E. N. Economou, "Elastic and acoustic wave band structure," J. Sound Vib., vol. 158, pp. 377-382, 1992.

[2] M. Torres, F. R. Montero de Espinosa, D. García-Pablos, and N. García, "Sonic band gaps in finite elastic media: Surface states and localization phenomena in linear and point defects," Phys. Rev. Lett., vol. 82, pp. 3054-3057, 1999.

[3] M. Kafesaki, M. Sigalas, and N. García, "Frequency modulation in the transmittivity of wave guides in elastic-wave band-gap materials," Phys. Rev. Lett., vol. 85, pp. 4044-4047, 2000.

[4] P. St. J. Russell, E. Marin, A. Díez, S. Guenneau, and A. B. Movchan, "Sonic band gaps in PCF performs: Enhancing the interaction of sound and light," Opt. Express, vol. 11, pp. 25552560, 2003.

[5] S. Yang, J. H. Page, Z. Liu, M. L. Cowan, C. T. Chan, and P. Sheng, "Focusing of sound in a 3D phononic crystal," Phys. Rev. Lett., vol. 93, pp. 024301: 1-4, 2004.

[6] Z.-G. Huang and T.-T. Wu, "Temperature effect on the bandgaps of surface and bulk acoustic waves in two-dimensional phononic crystals," IEEE Trans. Ultrason., Ferroelect., Freq. Contr., vol. 52, pp. 365-370, 2005.
[7] T.-T. Wu and Z.-G. Huang, "Level repulsion of bulk acoustic waves in composite materials," Phys. Rev. B, vol. 70, pp. 214304: $1-6,2004$

[8] Y. Tanaka and S. I. Tamura, "Surface acoustic waves in twodimensional periodic elastic structures," Phys. Rev. B, vol. 58, pp. 7958-7965, 1998.

[9] T.-T. Wu, Z.-G. Huang, and S. Lin, "Surface and bulk acoustic waves in two-dimensional phononic crystal consisting of materials with general anisotropy," Phys. Rev. B, vol. 69, pp. 094301: $1-10,2004$.

[10] T.-T. Wu, Z.-C. Hsu, and Z.-G. Huang, "Band gaps and the electromechanical coupling coefficient of a surface acoustic wave in a two-dimensional piezoelectric phononic crystal," Phys. Rev. $B$, vol. 71, pp. 064303: 1-5, 2005.

[11] M. Wilm, S. Ballandras, V. Laude, and T. Pastureaud, "A full 3D plane-wave-expansion model for 1-3 piezoelectric composite structures," J. Acoust. Soc. Amer., vol. 112, pp. 943-952, 2002.

[12] M. Kafesaki and E. N. Economou, "Multiple-scattering theory for three-dimensional periodic acoustic composites," Phys. Rev. B, vol. 60, pp. 11993-12001, 1999.

[13] I. E. Psarobas, N. Stefanou, and A. Modinos, "Scattering of elastic waves by periodic arrays of spherical bodies," Phys. Rev. $B$, vol. 62, pp. 278-291, 2000.

[14] M. M. Sigalas and C. M. Soukoulis, "Elastic-wave propagation through disordered and/or absorptive layered systems," Phys. Rev. B, vol. 51, pp. 2780-2789, 1995.

[15] D. García-Pablos, M. Sigalas, F. R. Montero de Espinosa, M. Torres, M. Kafesaki, and N. García, "Theory and experiments on elastic band gaps," Phys. Rev. Lett., vol. 84, pp. 4349-4352, 2000.

[16] Y. Tanaka, Y. Tomoyasu, and S. I. Tamura, "Band structure of acoustic waves in phononic lattices: Two-dimensional composites with large acoustic mismatch," Phys. Rev. B, vol. 62, pp. 7387$7392,2000$.

[17] J.-H. Sun and T.-T. Wu, "Analyses of mode coupling in joined parallel phononic crystal waveguides," Phys. Rev. B, vol. 71, pp. 174303: 1-8, 2005.

[18] T. Gorishnyy, C. K. Ullal, M. Maldovan, G. Fytas, and E. L. Thomas, "Hypersonic phononic crystals," Phys. Rev. Lett., vol. 94, pp. 115501: 1-4, 2005.

[19] J. O. Vasseur, P. A. Deymier, B. Chenni, B. Djafari-Rouhani, L. Dobrzynski, and D. Prevost, "Experimental and theoretical evidence for the existence of absolute acoustic band gaps in twodimensional solid phononic crystals," Phys. Rev. Lett., vol. 86, pp. 3012-3015, 2001.

[20] T.-T. Wu, L.-C. Wu, and Z.-G. Huang, "Frequency band-gap measurement of two-dimensional air/silicon phononic crystals using layered slanted finger interdigital transducers," J. Appl. Phys., vol. 97, pp. 094916: 1-7, 2005.

[21] O. Matsuda, O. B. Wright, D. H. Hurley, V. E. Gusev, and K. Shimizu, "Coherence shear phononic generation and detection with ultrasonic optical pulses," Phys. Rev. Lett., vol. 93, pp. 095501: 1-4, 2004.

[22] O. Matsuda, T. Tachizaki, T. Fukui, J. J. Baumberg, and O. B. Wright, "Acoustic phonon generation and detection in $\mathrm{GaAs} / \mathrm{Al}_{0.3} \mathrm{Ga}_{0.7} \mathrm{As}$ quantum wells with picosecond laser pulses," Phys. Rev. B, vol. 71, pp. 115330: 1-13, 2005.

[23] V. Laude, M. Wilm, S. Benchabane, and A. Khelif, "Full band gap for surface acoustic waves in a piezoelectric phononic crystal," Phys. Rev. E, vol. 71, pp. 036607: 1-7, 2005.

[24] J. L. Bleustein, "A new surface wave in piezoelectric materials," Appl. Phys. Lett., vol. 13, pp. 412-413, 1968.

[25] Y. V. Gulyaev, "Electroacoustic surface waves in solids," Sov. Phys. JETP Lett., vol. 9, pp. 63-65, 1969.

[26] Y. Ohta, K. Nakamura, and H. Shimizu, "Piezoelectric surface shear waves," in Proc. Ultrason. Committee Inst. Electron. Commun. Eng. Japan, Apr. 1969. (in Japanese)

[27] C. K. Campbell, Surface Acoustic Wave Devices for Mobile and Wireless Communications. San Diego: Academic, 1998.

[28] K. Hashimoto and M. Yamaguchi, "Excitation and propagation of shear-horizontal-type surface and bulk acoustic waves," IEEE Trans. Ultrason., Ferroelect., Freq. Contr., vol. 48, pp. 11811188, 2001.

[29] Y. V. Gulyaev and F. S. Hickernell, "Acoustoelectronics: History, present state, and new ideas for a new era," in Proc. IEEE Ultrason. Symp., 1998, pp. 935-938. 
[30] D. Royer and E. Dieulesaint, Elastic Waves in Solids I: Free and Guided Propagation. Berlin: Springer-Verlag, 2000.

[31] B. A. Auld, Acoustic Fields and Waves in Solids. 2nd ed. Malabar, FL: Krieger, 1990.

[32] K. A. Ingebrigtsen, "Surface waves in piezoelectrics," J. Appl. Phys., vol. 40, pp. 2681-2689, 1969.

[33] M. S. Kushwaha, P. Halevi, and G. Martinez, "Theory of acoustic band structure of periodic composites," Phys. Rev. B, vol. 49, pp. 2313-2322, 1994.

[34] Y. V. Gulyaev, "Review of shear surface acoustic waves in solids," IEEE Trans. Ultrason., Ferroelect., Freq. Contr., vol. 48, pp. 935-938, 1998.

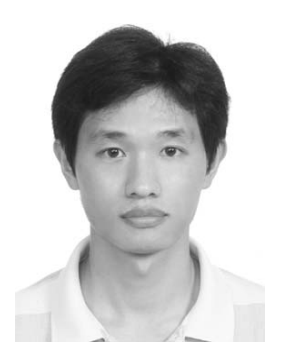

Jin-Chen Hsu was born in Changhua, Taiwan, in 1974. He received the B.S. degree in mechanical engineering from the National Taiwan University of Science and Technology in 1997. Currently, he is a Ph.D. candidate of the Institute of Applied Mechanics of National Taiwan University.

His research interests are in acoustic waves in piezoelectric media and phononic crystals.

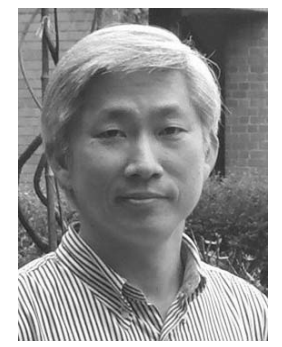

Tsung-Tsong $\mathbf{W u}$ received the doctorate in theoretical and applied mechanics from Cornell University in 1987. Then he joined the National Taiwan University faculty and currently is a professor of the Institute of Applied Mechanics of the National Taiwan University.

His interests are in ultrasonic wave propagation and related precision measurement techniques. Particular interests include RF ultrasonic sensing system, phononic crystals and frequency filter, and nondestructive evaluation of thin film.

Dr. Wu received the Distinguished Research Award of the National Science Council (NSC), Taiwan, three times for six years from 1995 to 2001 and is currently a distinguished research fellow of NSC. He is the executive board director of the Taiwanese Society of Nondestructive Testing, and the board director of the Quartz Industry Association of Taiwan. He is a fellow of the American Society of Mechanical Engineers. 